\title{
Efecto del pretratamiento con L-arginina en un modelo experimental de síndrome de insuficiencia respiratoria aguda inducido por ácido oleico
}

\author{
Effect of L-arginine pretreatment on an experimental model of \\ oleic acid-induced acute respiratory distress syndrome
}

\author{
Claudia Hernández-Jiménez, ${ }^{*}$ Matilde Baltazares-Lipp,* J Raúl Olmos-Zúñiga,* Miguel Gaxiola-Gaxiola,* \\ Axel Edmundo Guzmán-Cedillo, Mariana Silva-Martínez,* Rogelio Jasso-Victoria*
}

*Instituto Nacional de Enfermedades Respiratorias Ismael Cosío Villegas, Ciudad de México, México.

RESUMEN. Introducción: El síndrome de insuficiencia respiratoria aguda en el endotelio provoca vasoconstricción, adhesión de células inflamatorias y desarrollo de edema pulmonar, hipertensión pulmonar y trombosis. Este daño endotelial reduce la producción de óxido nítrico e incrementa la expresión de endotelina-1 (ET-1). Objetivo: En este estudio se evalúa el efecto del pretratamiento con L-arginina en la lesión pulmonar aguda inducida por ácido oleico. Material y métodos: Se analizaron los cambios hemodinámicos, gasométricos, microscópicos, inmunohistoquímicos para ET-1 y ETB y la concentración plasmática de ET-1 en un modelo experimental del síndrome de insuficiencia respiratoria aguda inducido por ácido oleico. Los perros fueron divididos en tres grupos de estudio: I $(n=6)$, control; II $(n=6)$ síndrome de insuficiencia respiratoria aguda, inducido por ácido oleico a una dosis IV de $0.09 \mathrm{~mL} /$ $\mathrm{kg}$, sin recibir tratamiento, y III $(\mathrm{n}=6)$, síndrome de insuficiencia respiratoria aguda inducido por ácido oleico y pretratamiento con L-arginina a $0.125 \mathrm{~g} / \mathrm{min} / \mathrm{IV}$ en $250 \mathrm{~mL}$ de solución salina $0.09 \%$. Resultados: Los grupos II y III desarrollaron síndrome de insuficiencia respiratoria agu-

\author{
Abreviaturas: \\ SIRA = Insuficiencia respiratoria aguda. \\ $\mathrm{ON}=$ Óxido nítrico. \\ ET-1 = Endotelina-1. \\ $\mathrm{AO}=$ Ácido oleico.
}

\section{Correspondencia:}

Dr. Rogelio Jasso-Victoria

Instituto Nacional de Enfermedades Respiratorias

Ismael Cosío Villegas, Ciudad de México, México.

Correo electrónico: rojavi_2000@yahoo.com

Trabajo recibido: 02-X-2020; aceptado: 24-XI-2020.

Citar como: Hernández-Jiménez C, Baltazares-Lipp M, Olmos-Zúñiga JR, Gaxiola-Gaxiola M, Guzmán-Cedillo AE, Silva-Martínez M et al. Efecto del pretratamiento con L-arginina en un modelo experimental de síndrome de insuficiencia respiratoria aguda inducido por ácido oleico. Neumol Cir Torax. 2020; 79 (4): 236-247. https://dx.doi.org/10.35366/97966
ABSTRACT. Introduction: Acute respiratory distress syndrome (ARDS) in the endothelium causes vasoconstriction, adhesion of inflammatory cells and development of pulmonary edema, pulmonary hypertension and thrombosis. This endothelial damage lowers the production of nitric oxide (NO) and increases the expression of endothelin-1 (ET-1). The aim of this study was to evaluate the effect of pretreatment with L-arginine in OA-induced ALI. Material and methods: Hemodynamic, gasometric, microscopic, immunohistochemical and plasma concentration of ET-1 and ET-1 y ETB were analyzed in an experimental model of AO-induced ARDS. The dogs were divided into three study groups: I $(n=6)$, control; II $(\mathrm{n}=6), \mathrm{OA}$-induced ARDS at an IV dose of $0.09 \mathrm{~mL} / \mathrm{kg}$, untreated, and III $(\mathrm{n}=6)$, pretreatment with L-arginine at $0.125 \mathrm{~g} / \mathrm{min} / \mathrm{IV}$ in $250 \mathrm{~mL}$ of $0.09 \%$ saline and OA-induced ARDS. Results: The hemodynamic, blood gas and gravimetric findings revealed that groups II and III developed ARDS. Intrapulmonary shunt increased in groups II and III $(p<0.05$, ANDEVA Dunnett). $\mathrm{PaO}_{2} / \mathrm{FiO}_{2}$ decreased in groups II and III with a significant difference with group I ( $p<0.05$, ANDEVA MR Dunnett).

$\mathrm{ET}_{\mathrm{B}}=$ Receptor tipo B.

ANDEVA MR = Análisis de la varianza de medidas repetidas.

aa $=$ Aminoácido.

$\mathrm{FiO}_{2}=$ Fracción inspirada de oxígeno

$\mathrm{GC}=$ Gasto cardíaco

RVP $=$ Resistencia vascular pulmonar.

RVS $=$ Resistencia vascular sistémica.

MAP $=$ Presión arterial media.

PMAP $=$ Presión media de la arteria pulmonar.

POAP $=$ Presión de oclusión de la arteria pulmonar.

$\mathrm{QS} / \mathrm{QT}=$ Cortocircuito intrapulmonar.

SVRi = Índice de resistencia vascular sistémica.

PVRi $=$ Índice de resistencia vascular pulmonar.

$\mathrm{PaO}_{2}=$ Presión de oxígeno arterial.

$\mathrm{HE}=$ Hematoxilina-eosina.

$\mathrm{PMN}=$ Polimorfonucleares

eNOS = Óxido nítrico sintetasa endotelial.

GMPc = Guanosín monofosfato cíclico.

LPS $=$ Lipopolisacárido.

iNOS = Óxido nítrico sintetasa inducible. 
da. El cortocircuito intrapulmonar se incrementó en los grupos II y III ( $p<0.05$, ANDEVA Dunnett). $\mathrm{La} \mathrm{PaO}_{2} / \mathrm{FiO}_{2}$ disminuyó en los grupos II y III con diferencia significativas con el grupo I $(p<0.05$, ANDEVA MR Dunnett). El grupo III mostró menos infiltrado inflamatorio y edema en el tejido pulmonar ( $p<0.05$ ANDEVA MR Dunnett). La expresión in situ de ET-1 y $\mathrm{ET}_{\mathrm{B}}$ fue mayor en el grupo II que en el grupo III. Los niveles plasmáticos de ET-1 mostraron un valor mayor en el grupo III $(p<0.05$ ANDEVA MR Dunnett). Conclusiones: El pretratamiento con L-arginina en un modelo experimental de SIRA agudo inducido por AO atenuó la vasoconstricción pulmonar hipóxica (HPV), lo que llevó a un deterioro en el cortocircuito intrapulmonar. Sin embargo, disminuyó el edema y el infiltrado inflamatorio en el tejido, por tanto, en este modelo puede considerarse únicamente un potencial efecto atenuante en la inflamación pulmonar durante la fase aguda por L-arginina.

Palabras clave: Edema pulmonar, donadores de óxido nítrico, endotelio pulmonar, vasoconstricción.

\section{INTRODUCCIÓN}

El endotelio pulmonar es un componente importante de la unidad alveolocapilar. Está implicado en el mantenimiento de la homeostasis de los vasos sanguíneos, donde se sintetizan sustancias biológicamente activas que regulan el tono vasomotor como el óxido nítrico (ON) y la endotelina (ET-1), las cuales modulan las interacciones célula-célula (neutrófilos-endotelio), previenen trombosis y promueven la proliferación celular. ${ }^{1}$ En el síndrome de insuficiencia respiratoria aguda (SIRA) disminuye la producción de ON, por lo que el endotelio pierde su capacidad antiagregante plaquetaria, anticoagulante y fibrinolítica, y pasa de tener efectos vasodilatadores a causar vasoconstricción, adhesión celular, inflamación, edema, hipertensión pulmonar y trombosis. ${ }^{2,3}$ El sustrato para la síntesis de ON es la L-arginina (2-amino-5-guanidino ácido pentanoico) un aminoácido (aa) no esencial bajo condiciones fisiológicas, pero que se convierte en un aa condicionalmente esencial bajo estrés, y desempeña un papel importante en el metabolismo intermediario y en la disponibilidad de arginina en el paciente crítico. ${ }^{4-6}$ Aunado a esto, la disminución en la producción de vasodilatadores propicia la sobreexpresión de ET-1, ${ }^{7}$ la cual a través del receptor tipo $\mathrm{B}\left(\mathrm{ET}_{\mathrm{B}}\right)$, provoca la infiltración de neutrófilos en la circulación y aumento en la permeabilidad vascular alveolar. ${ }^{8}$ Los receptores $\mathrm{ET}_{\mathrm{B}}$ se encuentran en las células endoteliales ${ }^{9}$ y median la vasodilatación a través de la liberación de ON y prostaciclina. Este subtipo de receptor también es responsable de la depuración de ET-1 circulante a través de endocitosis.

A pesar de que los efectos benéficos de la L-arginina, principalmente efectos antiinflamatorios y en el tono vasomotor, han sido descritos desde hace varias décadas, actualmente continúa un creciente interés en los posibles efectos terapéuticos de su suplementación, en particular en enfermedades con trastornos endoteliales, como los obser-
The group III showed less inflammatory infiltrate and edema in lung tissue. Plasma levels of ET-1 showed a higher value for group III. The in situ expression of ET-1 and ETB was higher for group II than for group III. Conclusions: Pretreatment with L-arginine in an experimental model of AO-induced acute SIRA attenuated hypoxic pulmonary vasoconstriction (HPV) leading to deterioration in intrapulmonary shunting. However, the edema and inflammatory infiltrate in the tissue decreased, therefore, in this model you can only consider a potential attenuating effect on lung inflammation during the acute phase by L-arginine.

Keywords: Pulmonary edema, nitric oxide donors, pulmonary endothelium, vasoconstriction.

vados en SIRA provocado por coronavirus 2 (SARS-CoV-2), debido a que la síntesis alterada de $\mathrm{ON}$ se considera una característica de un endotelio disfuncional. 2,10

Por lo que este estudio es el primero en plantear la hipótesis de que debido a que los niveles de ET-1 están regulados por la síntesis de ON, y puesto que la administración de L-arginina puede influir en la liberación de ET-1, conduciría a su vez a la prevención o atenuación de daño endotelial en SIRA inducido por ácido oleico (AO). Por ende, teniendo en cuenta las múltiples funciones de la ET-1, en este estudio se analizan los cambios en hemodinamia, gasometría, cambios microscópicos, detección inmunohistoquímica de la expresión de ET-1, $\mathrm{ET}_{\mathrm{B}}$ y en la concentración plasmática de ET-1 en un modelo experimental de SIRA en perros inducido por $\mathrm{AO}$.

Para estudiar este efecto se utilizó el modelo de AO debido a que está bien caracterizado y reproduce las lesiones patológicas de la fase inicial del SIRA originadas por alteración en la integridad de la membrana alveolar-capilar presentes en humanos. ${ }^{11-13}$

\section{MATERIAL Y MÉTODOS}

Este estudio se realizó en el Departamento de Cirugía Experimental del Instituto Nacional de Enfermedades Respiratorias (INER) Ismael Cosío Villegas. Se utilizaron 18 perros mestizos adultos sanos, con un peso entre 18 y 20 $\mathrm{kg}$. Se decidió utilizar como modelo animal al perro porque su hilio pulmonar es largo y de fácil acceso; mientras que en cerdos y ovejas el sistema digestivo tiene un mayor desarrollo y esto puede causar diferencias hemodinámicas significativas. Todos los animales fueron tratados de acuerdo con las especificaciones técnicas para el Cuidado y Uso de Animales de Laboratorio de la Norma Oficial Mexicana NOM-062-ZOO-1999 ${ }^{14}$ y la Guía para el Cuidado y Uso de Animales de Laboratorio de los Estados Unidos de América. ${ }^{15}$ Antes del experimento, los animales fueron confinados 
en jaulas individuales $(1.0 \mathrm{~m}$ de ancho $\times 3.5 \mathrm{~m}$ de largo $\times$ $2.7 \mathrm{~m}$ de alto $)^{14}$ con las mismas condiciones ambientales, con agua y comida ad libitum. Este protocolo fue revisado y aprobado por el Comité de Bioética del INER (número de protocolo: B12-06). El tamaño de la muestra se redujo de acuerdo con los principios de las técnicas experimentales propuestas por Balls ${ }^{16}$ y Kilkenny. ${ }^{17}$

Para su estudio, los animales fueron divididos en tres grupos: grupo I $(n=6)$ : control sin SIRA; grupo II $(n=6)$ : SIRA inducido por AO; y grupo III $(n=6)$ : SIRA inducido por AO con administración de L-arginina (Sigma-Aldrich, Alemania).

Los animales fueron anestesiados con clorhidrato de tiletamina y zolazepam (Zoletil 50, Virbac, México) $8 \mathrm{mg} / \mathrm{kg}$ IV, fentanilo (Janssen-Cilag, México) $0.02 \mathrm{mg} / \mathrm{kg}$ IV, atropina (Atropisa, Pisa, México) $0.02 \mathrm{mg} / \mathrm{kg}$ IV, acepromacina (Calmivet Vetoquinol, México) $0.02 \mathrm{mg} / \mathrm{kg}$ IV. Los signos vitales y parámetros hemodinámicos fueron evaluados por medio de un monitor para electrocardiografía (Datascope Passport, EUA). Los perros se colocaron en posición dorsoventral (supino) y se intubaron orotraquealmente (Endotracheal tube, Rush. Kamunting, Malaysia) y se conectaron a un ventilador de volumen (Harvard Apparatus, EUA), con un volumen corriente de $10 \mathrm{~mL} / \mathrm{kg}$ y frecuencia respiratoria de 20 rpm, fracción inspirada de oxígeno $\left(\mathrm{FiO}_{2}\right)$ 100\%. ${ }^{18,19}$ Para mantener la anestesia, se utilizó un vaporizador (Isotec 3 Ohmeda) con isoflurano 1.5\% (PISA Laboratorios, México). Mediante disección se colocaron catéteres vasculares en arteria y vena femorales (Cordis Corp, Johnson \& Johnson, CA, USA) y en vena yugular derecha un catéter Swan-Ganz 5 Fr (Edwards Lifesciences, Irvine Cal, USA), a través de los cuales se registraron los parámetros hemodinámicos sistémicos, pulmonares y se realizó toma de muestras sanguíneas. El gasto cardíaco (GC) e índices de resistencia vascular pulmonar (RVP) y sistémica (RVS) fueron calculados mediante computadora (Hemodynamic Profile Computer Spectramed model SP-1445, Oxnard, EE.UU.).

\section{Inducción del SIRA}

El grupo II y III recibieron a través del catéter en la vena femoral $0.09 \mathrm{~mL} / \mathrm{kg}$ de $\mathrm{AO}$ (ácido cis-9-octadecenoico; CAS 112-80-1, Sigma Aldrich, Alemania) emulsificado con etanol 1:1 y diluido a $10 \mathrm{~mL}$ de solución salina fisiológica, se administró con un flujo constante de $0.5 \mathrm{~mL} / \mathrm{min}$ durante 20 minutos. ${ }^{20}$ El grupo III recibió pretratamiento con 0.125 $\mathrm{g} / \mathrm{min} / \mathrm{IV}$ de L-arginina (CAS 74-79-3, Sigma-Aldrich, Alemania) en $250 \mathrm{~mL}$ de solución salina $0.09 \%$ en el intervalo entre 60 minutos antes y 60 minutos después de la infusión de AO. ${ }^{1}$ La tasa de infusión se mantuvo por debajo de los límites de hipervolemia. ${ }^{21}$

Se diseñó como un estudio agudo con una duración de 165 minutos y al concluir este tiempo los animales fueron sometidos a eutanasia con una sobredosis de pentobarbital sódico (Anestesal, Pfizer, México). ${ }^{14}$

\section{Evaluación}

\section{Hemodinamia y gasometría}

Se evaluaron los siguientes parámetros: GC; presión arterial media (MAP); presión media de la arteria pulmonar (PMAP); presión de oclusión de la arteria pulmonar (POAP); cortocircuito intrapulmonar (QS/QT); índice de resistencia vascular sistémica (SVRi) y pulmonar (PVRi); presión de oxígeno arterial $\left(\mathrm{PaO}_{2}\right)$.

\section{Radiográfica}

Se tomaron radiografías ventrodorsales basales a los 60 y 150 minutos después de la administración del AO. La presencia de opacidades se cuantificó de acuerdo con su extensión sobre una escala de 0 a 4: sin opacidades (grado 0), opacidades hasta 25\% (grado 1), opacidad de 26 a 50\% (grado 2), de 51 a 75\% (grado 3), y de 76 a 100\% (grado

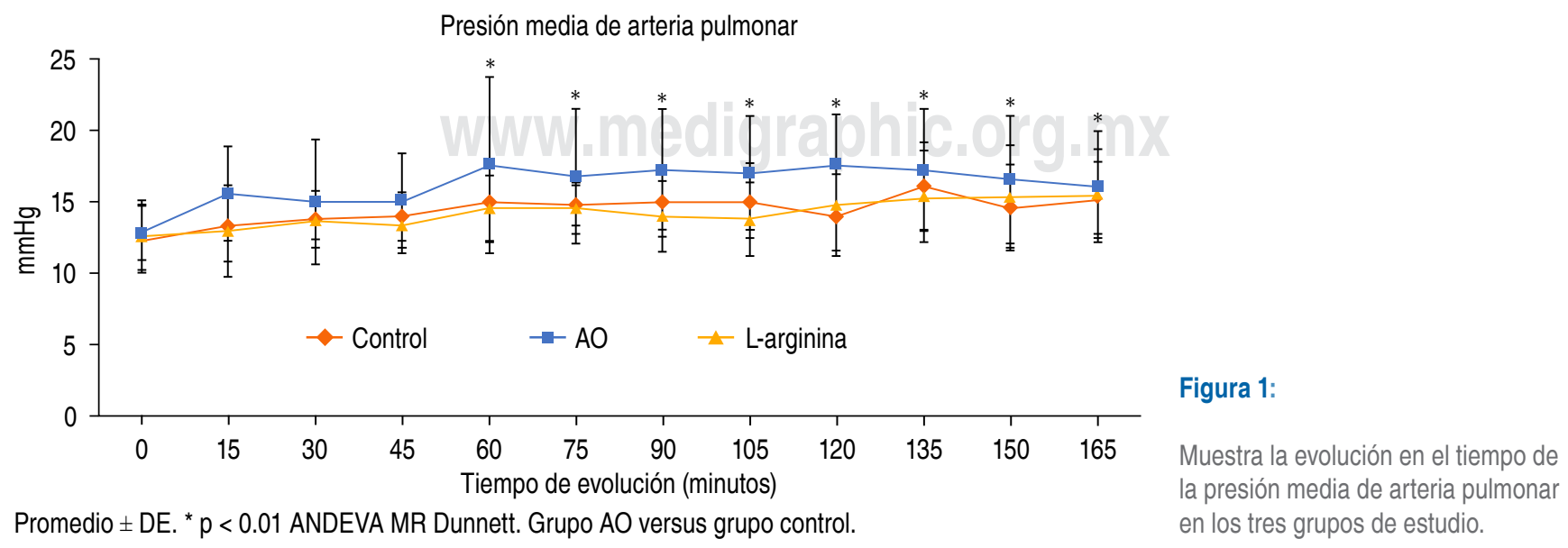


4). Dos investigadores determinaron el grado de manera independiente en un análisis doble ciego.

\section{Determinación del edema pulmonar}

El edema pulmonar se cuantificó por análisis gravimétrico. Los lóbulos pulmonares caudales se pesaron y secaron a una temperatura entre 60 y $65{ }^{\circ} \mathrm{C}$ hasta obtener peso constante. Finalmente, la ganancia de peso del pulmón se calculó mediante la siguiente fórmula: $\triangle \mathrm{PP}=(\mathrm{PH}-\mathrm{PS}) / \mathrm{PS}$, donde $\Delta \mathrm{PP}=$ ganancia de peso de pulmón, $\mathrm{PH}=$ peso final del pulmón y PS = peso inicial de pulmón.

\section{Evaluación microscópica}

El tejido pulmonar en todos los casos se tomó del lóbulo caudal, se fijó en formaldehído al 10\% y se embebió en parafina. Secciones de 4 micras de espesor, se tiñeron con hematoxilina-eosina (HE) y se analizaron por microscopia de luz para determinar la presencia de daño alveolar difuso caracterizado por edema, colapso alveolar, hemorragia, membranas hialinas, microtrombos e infiltración de neutrófilos. Los resultados se clasificaron en cuatro categorías: histología normal (grado 0), lesión leve (grado 1), lesión moderada (grado 2 ) y lesión grave (grado 3 ). ${ }^{22}$

\section{Cuantificación de ET-1}

Al final del estudio se obtuvieron muestras de sangre de la arteria pulmonar (catéter de Swan-Ganz) y se colocaron en tubos con EDTA, se centrifugaron a 1,800 rpm durante 15 minutos a $4{ }^{\circ} \mathrm{C}$ (CS-6R Beckman, EUA), posteriormente la muestra se concentró con una columna Sep-Pak C18 (Millipore, EUA). Se cuantificó con técnica de ELISA (Endothelin-1 ELISA kit, 900-020A, Enzo Life Sciences, EUA).

\section{Detección inmunohistoquímica de ET-1 y ETB}

La detección de la expresión in situ de ET-1 y ETB en el tejido pulmonar se realizó en secciones de 2 a 4 micras de espesor con anticuerpo anti-ET-1 monoclonal, MA3-005, Research Diagnostics, EUA) y anticuerpo Endothelin B receptor (Endothelin Receptor Polyclonal Antibody, 20RES002, Fitzgerald, EUA), con el sistema estreptavidinabiotina-peroxidasa (Vectastain Universal Quick Kit, Vector Laboratories, EUA). ${ }^{23}$ Para revelar se utilizó el cromógeno 3-amino-9-etil-carbazol (AEC) (Substrate Pack Biogenex, 932-HK092-F San Ramón CA, EUA). Se tiñeron con hematoxilina (Biocare Medical, CA, EUA) y para virar el contraste se utilizó carbonato de litio (Fisher chemical, NY, EUA). Las secciones fueron evaluadas semicuantitativamente por microscopia de luz de acuerdo con una escala de inmunotinción que describe proporción e intensidad. ${ }^{24}$

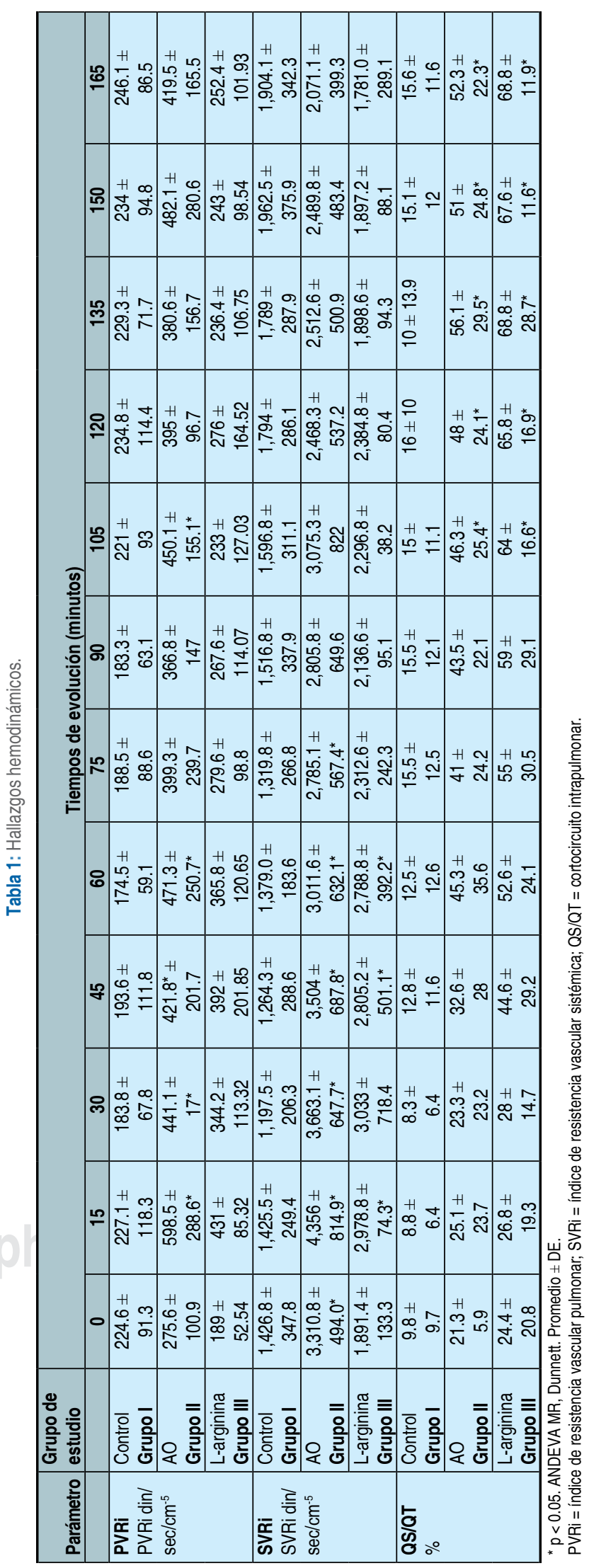


La puntuación se determinó sumando los resultados del porcentaje de células $(0=0 \% ; 1 \leq 30 \% ; 2=30-60 \%$ y $3 \geq 60 \%$ ) con el valor ordinal de intensidad de tinción ( 0 = negativo, 1 = débil, $2=$ medio y 3 = fuerte.) Así como la descripción de los diferentes componentes de tejido donde se expresó.

Los datos fueron expresados como media \pm EE. Para las variables de hemodinamia y gasometría se utilizó un análisis de la varianza de medidas repetidas (ANDEVA MR), para comparar contra el grupo control (ANDEVA, Dunnett) y con prueba de Bonferroni, cuando se detectó efecto entre sujetos. La prueba de normalidad que se utilizó fue ShapiroWilk ( $p<0.05)$; y la prueba de Levene para igualdad de varianzas $(p<0.05)$. Para el análisis radiográfico se realizó prueba de Wilcoxon $(p<0.05)$ para mediciones en el mismo grupo a lo largo del tiempo e índice kappa para estimar la concordancia entre observadores.

\section{RESULTADOS}

Todos los animales sobrevivieron al tiempo de estudio y los grupos II y III desarrollaron SIRA posterior a la administración del $\mathrm{AO}$.

\section{Hallazgos hemodinámicos y gasométricos}

Los parámetros hemodinámicos MAP, POAP y GC durante los 165 min del experimento permanecieron sin cambios significativos en todos los grupos ( $p>0.05$, ANDEVA MR). En el grupo II se observó incremento de la PMAP a partir de los 15 minutos posteriores a la administración de AO rebasando los límites de referencia. Sin embargo, a partir del minuto 90 se mostró un mayor incremento que se mantuvo hasta el final del estudio en comparación con el grupo control ( $p<0.05$, ANDEVA Dunnett) y el grupo III mostró valores menores de la PMAP en comparación al grupo I a partir del minuto 45 (P = NS, ANDEVA MR) (Figura 1).
La PVRi presentó diferencias significativas en el tiempo $(p=0.05$, ANDEVA MR) y al comparar entre grupos $(p=0.001$, ANDEVA MR Dunnett), con diferencia entre el grupo $\mathrm{AO}$ (control) y el grupo II (AO); por otra parte, el grupo III no difirió de los grupos I y II $(p=0.566$, ANDEVA MR Dunnett). Además, la SVRi presentó diferencias del grupo II (AO) versus el grupo I (control) $(p=0.05$, ANDEVA MR) (Tabla 1).

La $\mathrm{PaO}_{2}$ disminuyó a los 15 minutos después de la administración del AO en los grupos II (AO) y III (Larginina) por debajo de sus valores basales en comparación con el grupo I (control) durante todo el estudio. Si bien, la $\mathrm{PaO}_{2}$ fue menor en el grupo III (L-arginina) que en el II (AO) ( $p<0.01$, ANDEVA Dunnett) al final del estudio.

El cociente $\mathrm{PaO}_{2} / \mathrm{FiO}_{2}$ se utiliza para determinar la presencia del SIRA y de acuerdo al cociente se divide en: leve $\mathrm{PaO}_{2} / \mathrm{FiO}_{2} \leq 300$, pero $>200$; moderada $\mathrm{PaO}_{2} /$ $\mathrm{FiO}_{2} \leq 200$, pero $>100$; y severa $\mathrm{PaO}_{2} / \mathrm{FiO}_{2} \leq 100 .{ }^{25}$ En este estudio encontramos el desarrollo de SIRA en los grupos II (AO) y III (L-arginina), por la disminución de la relación $\mathrm{PaO}_{2} / \mathrm{FiO}_{2}$ a lo largo de los 165 min del experimento, con diferencia significativa en la relación $\mathrm{PaO}_{2} / \mathrm{FiO}_{2}$ de estos dos grupos con el grupo I (control) ( $p<0.05$, ANDEVA MR Dunnett) (Figura 2). Además, el QS/QT de los grupos II (AO) y III (L-arginina) tendió a aumentar significativamente después de $45 \mathrm{~min}$ de haber iniciado el experimento (Tabla 1).

\section{Análisis radiográfico}

Ningún animal en el grupo I (control) presentó cambios radiográficos. En el grupo II (AO) a los 60 minutos del estudio, tres animales mostraron opacidades alveolares bilaterales periféricas, difusas y asimétricas, con broncograma aéreo (grado 3); los tres animales restantes mostraron opacidades (grado 2), significativas estadísti-

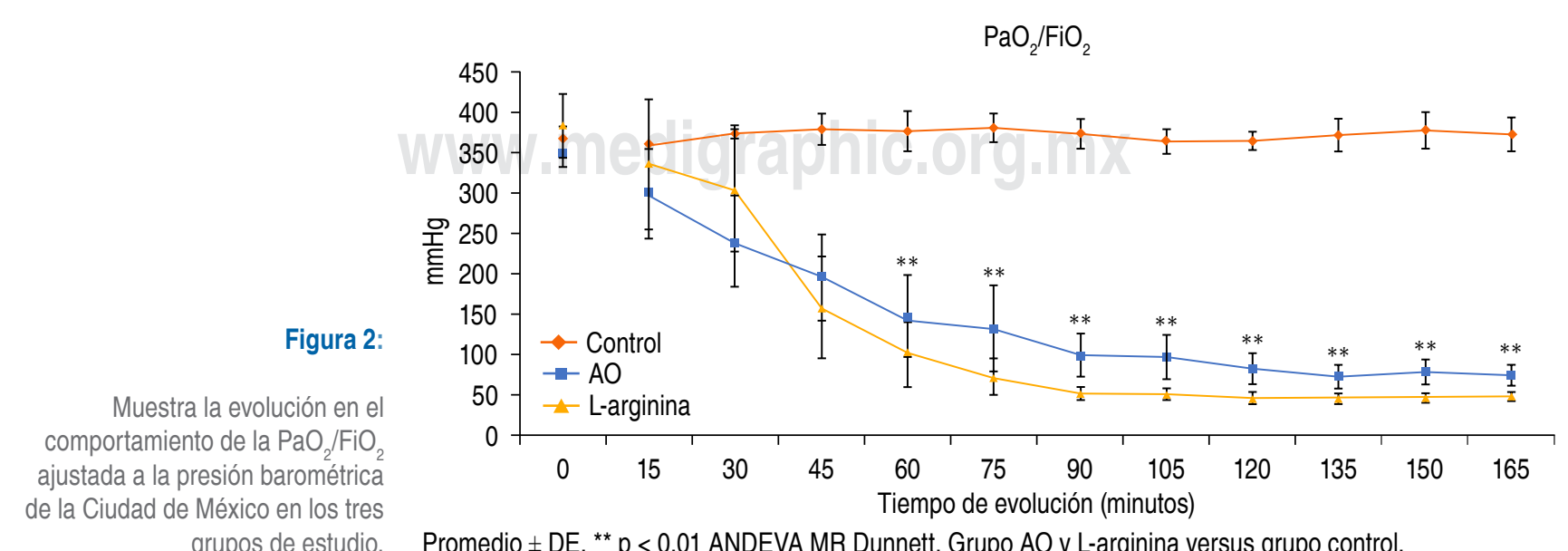

grupos de estudio.

Promedio \pm DE. ${ }^{* *} p<0.01$ ANDEVA MR Dunnett. Grupo AO y L-arginina versus grupo control. 
Neumol Cir Torax. 2020; 79 (4): 236-247
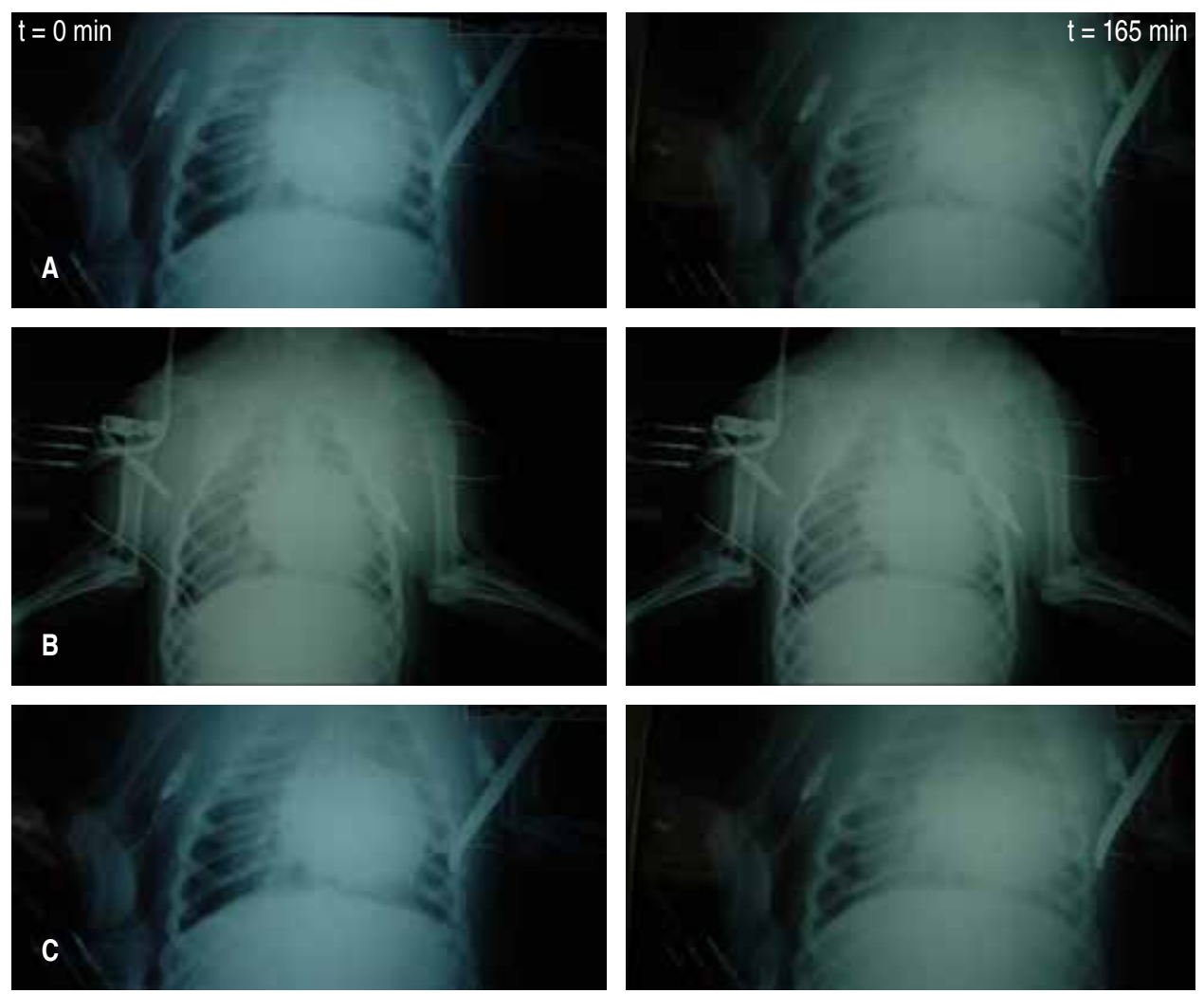

Figura 3:

Radiografías de tórax en proyección ventrodorsal que muestran: campos pulmonares del grupo I (control) A) basal y final; B) basal y final con infiltrados alveolares difusos en el grupo II (AO); y C) grupo III (L-arginina) basal y final con infiltrados alveolares difusos. camente versus radiografías basales y el grupo I (control) (Wilcoxon $z=-2.75, p<0.006$ ). Al final del estudio, en los grupos II (AO) y III (L-arginina) las opacidades fueron de grado 4 (Wilcoxon $z=3.002, p<0.003$ ). El índice Kappa arrojó un valor de 0.87 ( $p<0.001$ ), lo que indica un buen nivel de concordancia entre los observadores (Figura 3).

\section{Análisis gravimétrico}

El peso de los pulmones de los grupos de estudio II (AO) y III (L-arginina) aumentó (127.17 \pm 32.34 g y $107.00 \pm 29.06$ $\mathrm{g}$, respectivamente) en comparación con el grupo I $(42.08 \pm 13.26 \mathrm{~g}),(p<0.001$ ANDEVA MR, Dunnett). En la Figura 4 se presenta el peso húmedo/peso seco.

\section{Análisis histológico}

Histológicamente el grupo I (control) no presentó cambios. El grupo II (AO) desarrolló colapso alveolar (grado 2), edema alveolar (grado 3), hemorragia (grado 1), infiltración de polimorfonucleares (PMN) (grado 3), microtrombos (grado 2). El grupo III (L-arginina) presentó colapso alveolar (grado 2), edema (grado 2), hemorragia (grado 1), infiltración de PMN (grado 2), microtrombos (grado 1) $(p<0.05$ KruskalWallis) (Figura 5).

\section{Cuantificación de ET-1}

Los niveles plasmáticos de ET-1 mostraron diferencias significativas entre el grupo I (control) $(0.61 \pm 0.35 \mathrm{pg} / \mathrm{mL})$ y grupo II (AO) $(4.15 \pm 0.49 \mathrm{pg} / \mathrm{mL})(p<0.001$, ANDEVA Dunnett) y entre el grupo II (AO) y grupo III (L-arginina) $(5.5 \pm 2.50 \mathrm{pg} / \mathrm{mL})(\mathrm{p}<0.002$, ANDEVA Dunnett).

\section{Hallazgos inmunohistoquímicos}

La detección inmunohistoquímica de la expresión de ET-1 mostró en el grupo I (control) que 33\% de las muestras tuvo una positividad en porcentaje menor de $30 \%$, con una intensidad de tinción media. En el grupo II (AO) hubo positividad en $83.33 \%$ con una proporción menor de $30 \%$, con intensidad fuerte, y el grupo III (L-arginina) 100\% mostró una proporción menor de 30\% con una intensidad débil. Respecto al inmunomarcaje de $\mathrm{ET}_{\mathrm{B}^{\prime}}, 100 \%$ del grupo I (control) tuvo una proporción de tinción menor de 30\% con una intensidad media; el grupo II (AO) 100\% tuvo una proporción de tinción mayor de $60 \%$, con una intensidad fuerte, y en el grupo III (L-arginina) sólo 16.6\% de las muestras mostró una proporción en la tinción menor de 30\% con intensidad leve.

En ambos marcajes la tinción se observó principalmente en epitelio de bronquiolos y células endoteliales, seguida 
de las células epiteliales alveolares, en todos los casos con un patrón citoplasmático difuso (Figura 6).

\section{DISCUSIÓN}

En el presente trabajo se estudiaron los efectos de la administración de L-arginina en los cambios hemodinámicos, gasométricos, radiográficos, microscópicos, niveles plasmáticos de ET-1 y detección inmunohistoquímica para ET-1 y $\mathrm{ET}_{\mathrm{B}}$ durante el SIRA inducido por $\mathrm{AO}$, hipotetizando que debido a la disfunción endotelial, la administración de L-arginina antes de la inducción de SIRA podría influir en la liberación de ET-1, lo que a su vez conduciría a la prevención o atenuación de daño endotelial inducido por $\mathrm{AO}$.

Para esto se eligió el modelo de SIRA inducido por $\mathrm{AO}$, ya que desde su primera descripción por Ashbaugh et al. (1968), ${ }^{26}$ este modelo continúa vigente debido a que está bien caracterizado, ya que reproduce las lesiones patológicas de la fase inicial del SIRA presentes en humanos. ${ }^{10-12,27}$ La ventaja de este modelo frente a otros es que puede utilizarse en animales pequeños y grandes con la aparición aguda de las lesiones patológicas de SIRA después de la inyección de $\mathrm{AO}$, y la posibilidad de estudiarlo sin inflamación sistémica como en muchos otros modelos de sepsis. ${ }^{12}$ El mecanismo de acción que media la lesión pulmonar del AO ocurre por la unión directa de éste a las membranas biológicas, lo que provoca que se disparen las vías intracelulares que terminan en la muerte de las células pulmonares, es decir, es directamente tóxico para las células endoteliales, causando necrosis e induciendo congestión capilar y edema intersticial e intraalveolar. ${ }^{28-30} \mathrm{El} \mathrm{AO}$ induce principalmente necrosis, pero también provoca apoptosis por disminución del marcador antiapoptótico Bcl-2 y un aumento del marcador proapoptótico Bad. También activa las caspasas, aumentando la generación de especies reactivas de oxígeno e induce despolarización mitocondrial y apoptosis en leucocitos, ${ }^{31}$ por lo que no se requiere que las células inflamatorias o sus productos medien el daño. Tal como lo describen Matute-Bello et al. ${ }^{32}$ «existe una infiltración de neutrófilos, pero la lesión no depende de los polimorfonucleares». De tal forma que es un modelo que da la posibilidad de estudiar SIRA sin inflamación sistémica como en muchos otros modelos de sepsis, ${ }^{12}$ pero con el daño al parénquima pulmonar que lleva al aumento de la permeabilidad vascular, lo que a su vez induce acumulación de líquido extravascular, alteración del tono vascular pulmonar, y deterioro de la función pulmonar con cambios hemodinámicos concomitantes. Para la inducción de SIRA con $\mathrm{AO}$ en este estudio se empleó la técnica descrita por Hernández et al. ${ }^{20}$ y se pudo establecer en todos los grupos SIRA con sus consecuencias patológicas, cumpliendo con los lineamientos para establecer el diagnóstico según lo establece el Consenso de Berlín. ${ }^{25}$
En este trabajo, los grupos II (AO) y III (L-arginina) desarrollaron SIRA caracterizado por lesión de la membrana alveolocapilar, lo que causó edema pulmonar con incremento en la PMAP desde los 15 minutos posteriores a la administración de $\mathrm{AO}$, debido a que la inyección intravenosa de este ácido graso produce lesión endotelial, inflamación y daño al parénquima pulmonar, pero el incremento más notorio presente a los 90 minutos, posiblemente fue provocado por los cambios en la permeabilidad vascular pulmonar y a la presencia de edema pulmonar como lo describe Kluttig. ${ }^{33}$

El aumento del PVRi en el grupo II (AO) se debió al hecho de que SIRA inducido por AO provoca hipoxia y alteración de tono vascular. ${ }^{34,35}$ No obstante, en el grupo III (L-arginina) la administración exógena de L-arginina atenuó significativamente el incremento de la resistencia vascular, al ser un regulador crítico de la producción de ON endotelial vascular. Sin embargo, este aminoácido exclusivo precursor del $\mathrm{ON}$ endógeno en ausencia de lesión vascular, por lo regular no afecta la producción de $\mathrm{ON} .{ }^{2}$ Otros investigadores también han mostrado que la administración exógena de L-arginina atenúa la disfunción endotelial en diferentes modelos, como Abdullahi et al. ${ }^{36}$ quienes aluden que la suplementación oral de L-arginina mejoró la relajación dependiente del endotelio en ratas al mejorar la expresión del gen para óxido nítrico sintetasa endotelial (eNOS). Chao et al. ${ }^{37}$ también han reportado que la L-arginina puede atenuar la lesión pulmonar mediante la restauración de la función endotelial y disminución en la liberación de citocinas inflamatorias. Más recientemente también se conoce que las manifestaciones sistémicas observadas en el SIRA en la enfermedad por coronavirus (COVID-19), provocado por coronavirus 2 (SARS-CoV-2) pueden explicarse por una disfunción endotelial y por estos motivos, basándose en los efectos

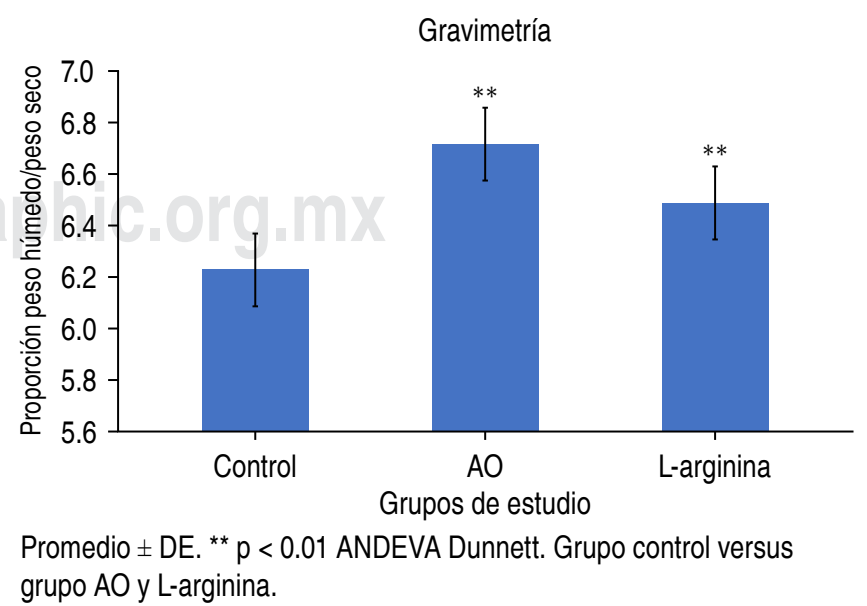

Figura 4: Peso del tejido pulmonar en todos los grupos de estudio. 
Figura 5:

Gráfica y micrografía de tejido
pulmonar. La gráfica muestra la escala
histopatológica con las medianas
para cada parámetro en cada grupo
(arriba). La micrografía (abajo)
exhibe la histología sin alteraciones
patológicas en septos, espacios
alveolares y vías aéreas del grupo
control; edema alveolar severo en el
grupo II (AO) e infiltración de neutrófilos
moderada, y grupo III (L-arginina)
sin alteraciones patológicas.

Gráfica y micrografía de tejido pulmonar. La gráfica muestra la escala histopatológica con las medianas para cada parámetro en cada grupo (arriba). La micrografía (abajo exhibe la histologia sin alteraciones
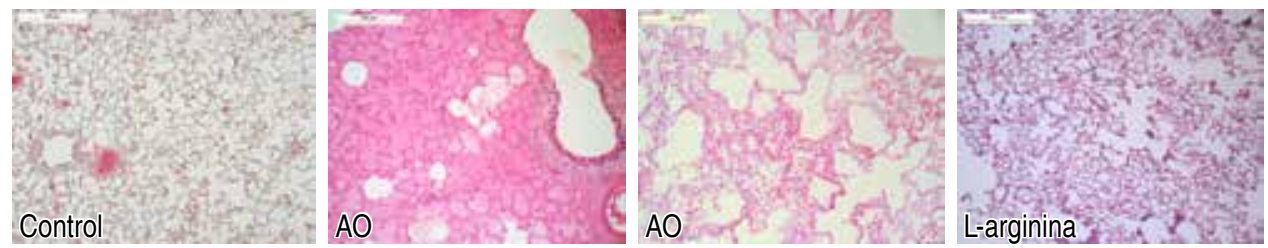

Colapso

Edema alveolar

Hemorragia

Membranas hialinas

Microtrombos

Congestión positivos de la L-arginina en la función endotelial, otros investigadores también especulan que la suplementación con arginina podría ser útil para contrastar la disfunción endotelial en pacientes con COVID-19. ${ }^{38,39} \mathrm{Al}$ ser la Larginina un aminoácido condicionalmente esencial en el paciente crítico la vía de administración no parece estar asociada con una alteración sustancial de los niveles disponibles de L-arginina. La biodisponibilidad relativa en el endotelio depende de que los mecanismos de síntesis de ON puedan estar saturados por la L-arginina endógena, también de que las células endoteliales y las células inflamatorias agudas como los macrófagos sean capaces de expresar arginasas que disminuyen localmente las reservas de L-arginina y privan de sustrato a la eNOS. Por tanto, el mecanismo potencial de la L-arginina implica la biodisponibilidad de la propia L-arginina o su inhibidor dimetilarginina asimétrica (ADMA). ${ }^{2}$

El aumento de QS/QT en los grupos II (AO) y III (Larginina) confirmó la presencia de edema, hemorragia y colapso alveolar, lo que impide el intercambio gaseoso. Sin embargo, el deterioro de QS/QT en el grupo III (L-arginina) en comparación con el grupo II (AO) sugiere que durante la hipoxia secundaria a SIRA, el ON liberado desde la célula endotelial atenuó la vasoconstricción pulmonar hipóxica (VPH). Se sabe que después de la administración de inhibidores del $\mathrm{ON}$ endógeno se mejora el intercambio gaseoso con redistribución, éste desde zonas hipóxicas a áreas bien ventiladas, lo que sugiere que el ON endógeno inhibe la VPH y puede agravar la relación ventilación/perfusión manteniendo el flujo sanguíneo en regiones deficientemente oxigenadas. El mecanismo molecular exacto de la VPH es menos conocido que la vasodilatación hipóxica en la circulación sistémica. Si bien, las células epiteliales alveolares pueden ser clave para detectar una baja concentración de oxígeno, y las células del músculo liso vascular pulmonar obviamente son las efectoras de la vasoconstricción, el endotelio vascular pulmonar desempeña un papel crucial como intermediario entre estos tipos de células. De hecho, Böger et al. ${ }^{40}$ observaron una liberación disfuncional de ON endotelial en humanos expuestos a hipoxia aguda, y los estudios en animales sugieren que la vasoconstricción pulmonar hipóxica se ve reforzada por la inhibición de la de NOS. Esto puede deberse, en parte a la elevación de ADMA, un inhibidor endógeno de la síntesis de $\mathrm{ON}$, ya que los niveles altos de ADMA se asocian con disfunción endotelial, enfermedad vascular e hipertensión.

La potenciación de la VPH por el ON se ha demostrado en diferentes modelos de alteración de la relación ventilación/perfusión intrapulmonar como la lesión pulmonar por AO. ${ }^{41}$ No obstante, la eficacia del ON depende del grado de alteración de la relación ventilación/perfusión predominante, siendo mayor en presencia de cortocircuito. Si el ON accede y vasodilata áreas con baja relación ventilación perfusión, la $\mathrm{PaO}_{2}$ puede aumentar, disminuir o no modificarse en absoluto. ${ }^{42}$ Pero la VHP también puede verse afectada por el incremento en la síntesis de ET-1 o la disminución en su depuración ${ }^{43}$ así como la disminución en la expresión de $\mathrm{ET}_{\mathrm{B}^{\prime}}$ ya que las endotelinas median la vasoconstricción pulmonar hipóxica y la hipoxia incrementa los niveles de endotelina en el plasma y los 
niveles de ARNm en el pulmón. El bloqueo selectivo y no selectivo de los receptores para ET-1 inhibe la HPV en algunos estudios, pero no en otros, esta discrepancia puede explicarse en parte por la liberación variable del $\mathrm{ON}$. Estos datos apoyan los hallazgos de este trabajo que sugieren que el ON endógeno atenuó la HPV y empeoró la relación ventilación/perfusión, lo que mantuvo el flujo sanguíneo en regiones mal oxigenadas.

La disminución en el peso de los pulmones en el grupo III (L-arginina) mostró que la administración aguda de L-arginina protegió los pulmones de incremento en la permeabilidad durante la lesión por AO, ya que se sabe que el $\mathrm{ON}$ es capaz de cambiar la presión de la microvasculatura, lo que puede resultar en la formación de edema, y además regula el balance de líquido en el pulmón. El mecanismo por el cual el ON estabiliza la permeabilidad microvascular todavía no es muy claro. Se sabe del efecto directo e indirecto del ON en el endotelio y epitelio, y puede actuar directamente sobre las uniones intercelulares por estimulación de GMPc (guanosín monofosfato cíclico), el cual causa relajación celular; hay evidencia de que esto puede estrechar las uniones intercelulares y disminuir la permeabilidad. ${ }^{44}$

Las alteraciones histopatológicas del SIRA inducido por AO encontradas en este trabajo están asociadas con lesión al endotelio pulmonar que lleva a evidentes alteraciones funcionales como la extravasación de líquido del espacio extravascular a los espacios aéreos como consecuencia del aumento de la permeabilidad de la barrera alveolocapilar. Este aumento de la permeabilidad se ve facilitado tanto por lesión como la activación del endotelio pulmonar, además la lesión epitelial conduce a una disminución de surfactante y por tanto, a una baja en el transporte de líquido. Asimismo, la presencia de infiltrado de polimorfonucleares en el grupo
Figura 6:

Inmunohistoquímica de tejido pulmonar (40x) con 3-amino-9-etilcarbazol (AEC). Muestra el inmunomarcaje en epitelio respiratorio para ET-1 medianamente positivo en: A) (I control); fuertemente positivo en B) (II AO) y C) (III L-arginina); y ETB medianamente positivo en D) (I control); fuertemente positivo en E) (II AO) y débilmente positivo en F) (III L-arginina).
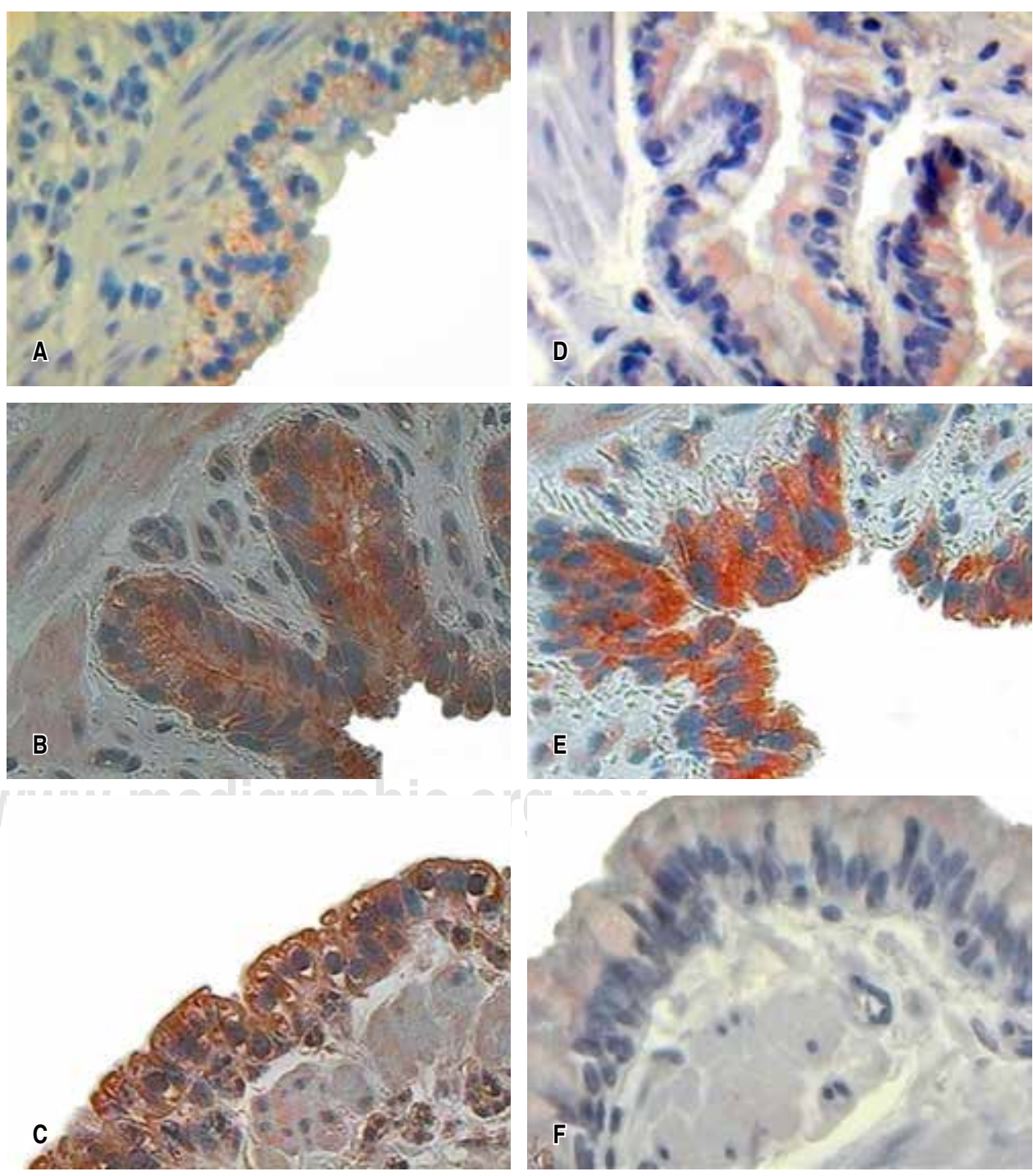
II (AO) reveló que el AO originó lesión endotelial y daño al parénquima pulmonar similar a la fase exudativa de SIRA. Aunque si bien el reclutamiento de neutrófilos en el pulmón es un sello distintivo de SIRA en modelos con lipopolisacárido (LPS), en el modelo de $\mathrm{AO}$ existe una infiltración de neutrófilos, pero la lesión no depende de PMN como lo describieron Matute et al..$^{32}$ Igualmente Huan-Ping Yu, et al. ${ }^{45}$ describieron que el AO puede tener efectos tanto favorables como desfavorables en el sistema inmunológico, ya que puede suprimir, mejorar o sinergizar la hiperactividad de los neutrófilos dependiendo de la dosis aplicada. Por otra parte, Guimaraes ${ }^{8}$ mostró que el SIRA inducido por AO activa al sistema ET-1, vía activación de receptores ETB, lo cual también puede influir en la infiltración de neutrófilos circulantes y en el aumento de la permeabilidad vascular alveolar como ocurrió en el grupo II (AO).

Los hallazgos histopatológicos en el grupo III demostraron que la administración de L-arginina disminuyó la infiltración de polimorfonucleares asociada con lesión vascular, ${ }^{46}$ lo cual concuerda con Wu et al. ${ }^{47}$ quienes estudiaron los efectos de la L-arginina en la respuesta inflamatoria, después de desafiar células epiteliales con LPS demostraron que un mecanismo de acción antiinflamatorio de la L-arginina es inhibir las vías de señalización de NF-kB. Además, la arginina también fue capaz de mejorar la $\beta$-caseína y la expresión de caseína total en células epiteliales mamarias bovinas inducidas por LPS.

El incremento en la concentración de ET-1 en los grupos II y III a partir del minuto 15 pos SIRA concuerda con trabajos previos, donde se ha observado que hay un aumento en la circulación en el SIRA tanto clínico como experimental y correlaciona con su severidad, ${ }^{48-50}$ y se sabe que la mejoría clínica correlaciona con la disminución de los niveles de ET-1. No obstante, el acrecentamiento de ET-1 en SIRA parece ser ocasionado más por un incremento en la síntesis pulmonar en la disminución de su depuración. En pacientes que murieron por SIRA se observó un incremento en la expresión de ET-1 en el endotelio vascular, músculo liso, epitelio de la vía aérea y macrófagos alveolares en comparación con pacientes que no murieron por SIRA y curiosamente estos pacientes tuvieron una disminución en la expresión de eNOS y óxido nítrico sintetasa inducible (iNOS) en el pulmón. ${ }^{51}$ Esta elevación de ET-1 también está relacionada con el incremento significativo en RVPi, ya que la ET-1 tiene un papel importante en el desarrollo de la vasoconstricción porque el lecho vascular es muy sensible a los efectos vasoconstrictores de ET-1 y ésta potencia el efecto vasoconstrictor de la activación simpática y su mismo efecto vasoconstrictor está potenciado por una disminución en la síntesis de $\mathrm{ON}$. Asimismo, la disminución de la relación $\mathrm{PaO}_{2} / \mathrm{FiO}_{2}$, como la que se presentó en los grupos de AO y L-arginina, y la progresión del QS/QT pueden estar asociadas al incremento en la concentración plasmática de ET-1. Del mismo modo, es posible que la disminución de la expresión de receptores B haya causado una disminución en la depuración de ET-1.

Debido a que los niveles de ET-1 plasmática así como el inmunomarcaje de ET-1 en el tejido pulmonar aumentaron significativamente, además de que $\mathrm{ET}_{\mathrm{B}}$ disminuyó en el grupo en el grupo III, L-arginina sugiere que hubo una regulación negativa de los receptores $\mathrm{ET}_{\mathrm{B}}$ in vivo inducida por ET-1. La posible explicación es que la disfunción endotelial que ocurre en el pulmón congestivo, puede resultar en la disminución de la expresión de $\mathrm{ET}_{\mathrm{B}^{\prime}}$ ya que el receptor existe principalmente en las células endoteliales, si éstas degeneran con rapidez, se espera entonces que los niveles de $\mathrm{ARNm}$ del receptor $\mathrm{ET}_{\mathrm{B}}$ disminuyan. Carpenter et al..$^{52}$ mostraron datos similares y llegaron a la conclusión de que los animales con deficiencia del receptor $\mathrm{ET}_{\mathrm{B}}$ tenían fuga de proteína vascular pulmonar exagerada en la normoxia, que la hipoxia exacerba esa fuga y que este efecto es en parte atribuible a un aumento mediado por ET-1.

Por ende, los resultados del presente estudio sugieren que el marcado incremento de ET-1 plasmática y por determinación inmunohistoquímica en el tejido pulmonar en el grupo III (L-arginina) puede ser atribuible a la disminución del aclaramiento por los receptores $\mathrm{ET}_{\mathrm{B}^{\prime}}$ pero también el aumento en los niveles de ET-1 influye en la progresión de la enfermedad pulmonar.

\section{CONCLUSIONES}

El pretratamiento con L-arginina en un modelo experimental de SIRA inducido por AO atenuó la VPH, disminuyó la infiltración inflamatoria en el tejido pulmonar, y puede proteger a los pulmones del incremento en la permeabilidad vascular. Por tanto, puede considerarse un posible papel terapéutico de la L-arginina en el edema e inflamación pulmonar.

\section{Limitaciones}

Es necesario considerar la existencia de limitaciones derivadas de un estudio de este tipo situado en el ámbito de la investigación. Se eligió un modelo de SIRA inducido por AO que ya ha sido empleado con anterioridad con buenos resultados, pero sabemos que no es una representación perfecta del SIRA clínico. Ambas condiciones tienen muchas similitudes, pero el AO causa la lesión directamente sin requerir que células inflamatorias o sus productos medien el daño, mientras que el SIRA está relacionado, la mayoría de las veces, con sepsis e inflamación.

Otra limitación a destacar es la asistencia mecánica ventilatoria, ya que ésta representa un componente primordial en el manejo del SIRA, lo que deriva en la dificultad de trasladar estos resultados a humanos. 


\section{Agradecimientos}

Los autores agradecen a Emilio R. Rueda Juárez y a Sergio Martínez Fonseca su asistencia en el cuidado de los animales.

\section{REFERENCIAS}

1. Piatti PM, Fragasso G, Monti LD, Setola E, Lucotti P, Fermo I. Acute intravenous L-arginine infusion decreases endothelin-1 levels and improves endothelial function in patients with angina pectoris and normal coronary arteriograms: correlation with asymmetric dimethylarginine levels. Circulation. 2003;107(3):429-436. https://doi.org/10.1161/01. cir.0000046489.24563.79

2. Cyr AR, Huckaby LV, Shiva SS, Zuckerbraun BS. Nitric oxide and endothelial dysfunction. Crit Care Clin. 2020;36(2):307-321. https:// doi.org/10.1016/j.ccc.2019.12.009

3. Guzmán Cedillo AE, Olmos Zúñiga JR, Jasso Victoria R, García Torrentera R, Gaxiola Gaxiola M, Silva Martínez M, et al. Efecto de tres modos ventilatorios como único soporte en un modelo experimental de inflamación sistémica por lipopolisacárido sobre la hemodinamia, fisiología pulmonar e histología. Neumol Cir Torax. 2020;79(1):37-49. doi: 10.35366/93428.

4. Luiking YC, Ten Have GA, Wolfe RR, Deutz NE. Arginine de novo and nitric oxide production in disease states. Am J Physiol Endocrinol Metab. 2012;303(10):E1177-E1189. https://doi.org/10.1152/ ajpendo.00284.2012

5. Rosenthal DM, Carrott WP, Patel J, Kiraly L, Martindale RG. Parenteralor enteral arginine supplementation safety and efficacy. J Nutr. 2016;146(12):2594S-600S. https://doi.org/10.3945/jn.115.228544

6. Sims CA, Holena D, Kim P, Pascual J, Smith B, Martin N, et al. Effect of low-dose supplementation of arginine vasopressin on need for blood product transfusions in patients with trauma and hemorrhagic shock: a randomized clinical trial. JAMA Surg. 2019;154(11):994-1003. https://doi.org/10.1001/jamasurg.2019.2884

7. Barton M, Yanagisawa M. Endothelin: 30 years from discovery to therapy. Hypertension. 2019;74(6):1232-1265. https://doi.org/10.1161/ hypertensionaha. 119.12105

8. Guimarães CL, Trentin PG, Rae GA. Endothelin ET(B) receptormediated mechanisms involved in oleic acid-induced acute lung injury in mice. Clin Sci (Lond). 2002;103 Suppl 48:340S-344S. https://doi. org/10.1042/cs103s340s

9. Hernández JC, OImos ZJR, Jasso VR, Baltazares LM, Gaxiola GM, Villalba CJ. Expresión de endotelina-1 y receptores para endotelina ETA y ETB en arteria pulmonar en un modelo de daño pulmonar agudo. Neumol Cir Torax. 2010;69(2):97-102.

10. Chen J, Zhang J, Shaik NF, Yi B, Wei X, Yang XF, et al. The histone deacetylase inhibitor tubacin mitigates endothelial dysfunction by up-regulating the expression of endothelial nitric oxide synthase. JBiol Chem. 2019;294(51):19565-19576. https://doi.org/10.1074/jbc.ra119.011317

11. Yan X, Li Y, Choi YH, Wang C, Piao Y, Ye J, et al. Protective effect and mechanism of alprostadil in acute respiratory distress syndrome induced by oleic acid in rats. Med Sci Monit. 2018;24:7186-7198. https://doi.org/10.12659/msm.909678

12. Kamuf J, Garcia-Bardon A, Ziebart A, Thomas R, Rümmler R, Möllmann $\mathrm{C}$, et al. Oleic acid-injection in pigs as a model for acute respiratory distress syndrome. J Vis Exp. 2018;(140):57783. doi: $10.3791 / 57783$.
13. Pechulis R, Satti AG, Wang P, Xue-Bing Q, Criner GJ. Acute lung injury (ALI) and acute respiratory distress syndrome (ARDS). In: Criner G, Barnette R, D'Alonzo G, editors. Critical care study guide. New York: Springer; 2010. Available from: https://doi.org/10.1007/978-0387-77452-7_16

14. Norma Oficial Mexicana NOM-062-ZOO-1999 [en línea]. Especificaciones técnicas para la producción, cuidado y uso de animales de laboratorio. Diario Oficial de la Federación; 1999. Accesible en: https://www.fmvz.unam.mx/fmvz/principal/archivos/062ZOO.PDF

15. National Research Council (US) Committee for the Update of the Guide for the Care and Use of Laboratory Animals. Guide for the care and use of laboratory animals. 8th ed. Washington (DC): National Academies Press (US); 2011. Available from: https://www.ncbi.nlm. nih.gov/books/NBK54050/doi: 10.17226/12910

16. Balls M. Replacement of animal procedures: alternatives in research, education and testing. Lab Anim. 1994;28(3):193-211. https://doi. org/10.1258/002367794780681714

17. Kilkenny C, Browne WJ, Cuthill IC, Emerson M, Altman DG. Improving bioscience research reporting: the ARRIVE guidelines for reporting animal research. PLoS Biol. 2010;8(6):e1000412. https:// doi.org/10.1371/journal.pbio.1000412

18. Bumbacher S, Schramel JP, Mosing M. Evaluation of three tidal volumes $(10,12$ and $15 \mathrm{~mL} \mathrm{~kg}-1)$ in dogs for controlled mechanical ventilation assessed by volumetric capnography: a randomized clinical trial. Vet Anaesth Analg. 2017;44(4):775-784. https://doi.org/10.1016/j. vaa.2016.10.007

19. Donati PA, Plotnikow G, Benavides G, Belerenian G, Jensen M, Londoño $L$. Tidal volume in mechanically ventilated dogs: can human strategies be extrapolated to veterinary patients? J Vet Sci. 2019;20(3):e21. https://doi.org/10.4142/jvs.2019.20.e21

20. Hernández-Jiménez C, Jasso-Victoria R, Olmos-Zúniga R, VillalbaCaloca J, Gaxiola-Gaxiola M, Sotres-Vega A, et al. Experimental model of canine oleic acid-induced acute lung injury: establishing a working model. Rev Invest Clin. 2010;62(3):222-230.

21. Valverde A, Gianotti G, Rioja-Garcia E, Hathway A. Effects of highvolume, rapid-fluid therapy on cardiovascular function and hematological values during isoflurane-induced hypotension in healthy dogs. Can J Vet Res. 2012;76(2):99-108.

22. Koksel O, Kaplan MB, Ozdulger A, Tamer L, Degirmenci U, Cinel $L$, et al. Oleic acid-induced lung injury in rats and effects of caffeic acid phenyl ester. Exp Lung Res. 2005;31(5):483-496. https://doi. org/10.1080/01902140590918876

23. Hsu SM, Raine L. Protein A, avidin, and biotin in immunohistochemistry. J Histochem Cytochem. 1981;29(11):1349-1353. https://doi. org/10.1177/29.11.6172466

24. Fedchenko N, Reifenrath J. Different approaches for interpretation and reporting of immunohistochemistry analysis results in the bone tissue-a review. Diagn Pathol. 2014;9:221. https://doi.org/10.1186/ s13000-014-0221-9

25. Barreira ER, Munoz GO, Cavalheiro PO, Suzuki AS, Degaspare NV, Shieh HH, et al; Brazilian Pediatric Acute Respiratory Distress Syndrome Study Group. Epidemiology and outcomes of acute respiratory distress syndrome in children according to the Berlin definition: a multicenter prospective study. Crit Care Med. 2015;43(5):947-953. https://doi.org/10.1097/ccm.0000000000000866

26. Ashbaugh DG, Uzawa T. Respiratory and hemodynamic changes after injection of free fatty acids. J Surg Res. 1968;8(9):417-423. https:// doi.org/10.1016/0022-4804(68)90057-7 
27. Carrillo Esper R, Sánchez Zúñiga MJ, Medveczky Ordoñes NI, Elizondo Argueta S, Ramírez Ambriz PM, Sánchez Pérez H. Síndrome de insuficiencia respiratoria aguda, 50 años después. Med Crit. 2017;31(4):246-254.

28. Gonçalves-de-Albuquerque CF, Silva RA, Burth P, de Moraes IM, Oliveira FM, Younes-Ibrahim M, et al. Oleic acid induces lung injury in mice through activation of the ERK pathway. Mediators Inflamm. 2012;2012:956509. https://doi.org/10.1155/2012/956509

29. Beilman G. Pathogenesis of oleic acid-induced lung injury in the rat: distribution of oleic acid during injury and early endothelial cell changes. Lipids. 1995;30(9):817-823. https://doi.org/10.1007/bf02533957

30. Gonçalves-de-Albuquerque CF, Burth P, Silva AR, de Moraes IM, de Jesus Oliveira FM, Santelli RE, et al. Oleic acid inhibits lung Na/KATPase in mice and induces injury with lipid body formation in leukocytes and eicosanoid production. J Inflamm (Lond). 2013;10(1):34. https://doi.org/10.1186/1476-9255-10-34

31. Gonçalves-de-Albuquerque CF, Silva AR, Burth P, Castro-Faria MV, Castro-Faria-Neto HC. Acute respiratory distress syndrome: role of oleic acid-triggered lung injury and inflammation. Mediators Inflamm. 2015;2015:260465. https://doi.org/10.1155/2015/260465

32. Matute-Bello G, Frevert CW, Martin TR. Animal models of acute lung injury. Am J Physiol Lung Cell Mol Physiol. 2008;295(3):L379-L399. https://doi.org/10.1152/ajplung.00010.2008

33. Kluttig R, Friedheim T, Behem C, Zach N, Brown R, Graessler M, et al. Invasive hemodynamic monitoring of aortic and pulmonary artery hemodynamics in a large animal model of ARDS. J Vis Exp. 2018;(141). https://doi.org/10.3791/57405

34. Kafi SA, Scillia P, Mélot C, Gevenois PA, Pagnamenta A, Naeije R. Abnormal pulmonary vascular tone in canine oleic acid lung injury. Crit Care Med. 2002;30(7):1565-1569. https://doi.org/10.1097/00003246200207000-00028

35. Leeman M, Lejeune P, Closset J, Vachiéry JL, Mélot C, Naeije R. Nature of pulmonary hypertension in canine oleic acid pulmonary edema. J Appl Physiol (1985). 1990;69(1):293-298. https://doi.org/10.1152/ jappl.1990.69.1.293

36. Adejare A, Oloyo A, Anigbogu C, Jaja S. L-arginine supplementation increased only endothelium-dependent relaxation in sprague-dawley rats fed a high-salt diet by enhancing abdominal aorta endothelial nitric oxide synthase gene expression. Clin Med Insights Cardiol. 2020;14:1179546820902843. https://doi. org/10.1177/1179546820902843

37. Chao YK, Wu YC, Yang KJ, Chiang LL, Liu HP, Lin PJ, et al. Pulmonary perfusion with L-arginine ameliorates post-cardiopulmonary bypass lung injury in a rabbit model. J Surg Res. 2011;167(2):e77-e83. https:// doi.org/10.1016/j.jss.2009.10.041

38. Sardu C, Gambardella J, Morelli MB, Wang X, Marfella R, Santulli G. Hypertension, thrombosis, kidney failure, and diabetes: is COVID-19 an endothelial disease? A comprehensive evaluation of clinical and basic evidence. J Clin Med. 2020;9(5):1417. https://doi.org/10.3390/ jcm9051417

39. Gambardella J, Khondkar W, Morelli MB, Wang X, Santulli G, Trimarco V. Arginine and endothelial function. Biomedicines. 2020;8(8):277. https://doi.org/10.3390/biomedicines8080277

40. Böger R, Hannemann J. Dual role of the L-arginine-ADMA-NO pathway in systemic hypoxic vasodilation and pulmonary hypoxic vasoconstriction. Pulm Circ. 2020;10(2):2045894020918850. https:// doi.org/10.1177/2045894020918850
41. Putensen C, Räsänen J, Downs JB. Effect of endogenous and inhaled nitric oxide on the ventilation-perfusion relationships in oleic-acid lung injury. Am J Respir Crit Care Med. 1994;150(2):330-336. https://doi. org/10.1164/ajrccm.150.2.8049811

42. Dunham-Snary KJ, Wu D, Sykes EA, Thakrar A, Parlow LRG, Mewburn JD, et al. Hypoxic pulmonary vasoconstriction: from molecular mechanisms to medicine. Chest. 2017;151(1):181-192. https:// doi.org/10.1016/j.chest.2016.09.001

43. Kelland NF, Bagnall AJ, Morecroft I, Gulliver-Sloan FH, Dempsie Y, Nilsen $\mathrm{M}$, et al. Endothelial ET(B) limits vascular remodelling and development of pulmonary hypertension during hypoxia. J Vasc Res. 2010;47(1):16-22. https://doi.org/10.1159/000231717

44. Durán WN, Breslin JW, Sánchez FA. The NO cascade, eNOS location, and microvascular permeability. Cardiovasc Res. 2010;87(2):254-261. https://doi.org/10.1093/cvr/cvq139

45. Yu HP, Liu FC, Umoro A, Lin ZC, Elzoghby AO, Hwang TL, et al. Oleic acid-based nanosystems for mitigating acute respiratory distress syndrome in mice through neutrophil suppression: how the particulate size affects therapeutic efficiency. J Nanobiotechnology. 2020;18(1):25. https://doi.org/10.1186/s12951-020-0583-y

46. Qiu Y, Yang X, Wang L, Gao K, Jiang Z. L-arginine inhibited inflammatory response and oxidative stress induced by lipopolysaccharide via arginase-1 signaling in IPEC-J2 cells. Int J Mol Sci. 2019;20(7):1800. https://doi.org/10.3390/ijms20071800

47. Wu T, Wang C, Ding L, Shen Y, Cui H, Wang M, et al. Arginine relieves the inflammatory response and enhances the casein expression in bovine mammary epithelial cells induced by lipopolysaccharide. Mediators Inflamm. 2016;2016:9618795. https://doi. org/10.1155/2016/9618795

48. Araz O. Current pharmacological approach to ARDS: The place of bosentan. Eurasian J Med. 2020;52(1):81-85. https://doi.org/10.5152/ eurasianjmed.2020.19218

49. Jesmin S, Yamaguchi N, Zaedi S, Sultana SN, Iwashima Y, Sawamura $A$, et al. Time-dependent expression of endothelin-1 in lungs and the effects of TNF- $\alpha$ blocking peptide on acute lung injury in an endotoxemic rat model. Biomed Res. 2011;32(1):9-17. https://doi. org/10.2220/biomedres.32.9

50. Comellas AP, Briva A. Role of endothelin-1 in acute lung injury. Transl Res. 2009;153(6):263-271. https://doi.org/10.1016/j.trsl.2009.02.007

51. Albertine KH, Wang ZM, Michael JR. Expression of endothelial nitric oxide synthase, inducible nitric oxide synthase, and endothelin-1 in lungs of subjects who died with ARDS. Chest. 1999;116(1 Suppl):101S$102 S$.

52. Carpenter T, Schomberg S, Steudel W, Ozimek J, Colvin K, Stenmark $\mathrm{K}$, et al. Endothelin B receptor deficiency predisposes to pulmonary edema formation via increased lung vascular endothelial cell growth factor expression. Circ Res. 2003;93(5):456-463. https://doi. org/10.1161/01.res.0000090994.15442.42

Financiamiento: La investigación que lleva a estos resultados ha sido financiada por el Instituto Nacional de Enfermedades Respiratorias Ismael Cosío Villegas.

Conflicto de intereses: Los autores declaran no tener conflicto de intereses. 